\title{
Rectal Fistula, CTCAE
}

National Cancer Institute

\section{Source}

National Cancer Institute. Rectal Fistula, CT CAE. NCI Thesaurus. Code C57859.

A disorder characterized by an abnormal communication between the rectum and another organ or anatomic site. 